\title{
APPLICATIONS OF ACOUSTICS AND CAVITATION TO NON-INVASIVE THERAPY AND DRUG DELIVERY
}

\author{
Constantin-C. Coussios ${ }^{1}$ and Ronald A Roy ${ }^{2}$
}

\footnotetext{
${ }^{1}$ Institute of Biomedical Engineering, Department of Engineering Science, University of Oxford, Oxford OX1 3PJ, UK; email: constantin.coussios@eng.ox.ac.uk

${ }^{2}$ Department of Aerospace and Mechanical Engineering, Boston University, Boston, MA 02215, USA; email: ronroy@bu.edu
}

Corresponding Author:

Dr Constantin Coussios

Institute of Biomedical Engineering

Department of Engineering Science

17 Parks Road

Oxford OX1 3PJ

UK

Tel: +44-1865-274750

Fax: +44-1865-274752

Email: constantin.coussios@eng.ox.ac.uk 


\begin{abstract}
Biomedical acoustics is rapidly evolving from a diagnostic modality into a therapeutic tool and acoustic cavitation is often found to be the common denominator in a wide range of new therapeutic applications. High-intensity focussed ultrasound (HIFU) waves generated outside the body can be used to deposit heat deep within the body. Through a quantitative analysis of heat deposition by ultrasound, it is shown that inertial cavitation can help address some of the major challenges of HIFU therapy by providing a means of enhancing and monitoring treatment non-invasively. In the context of drug delivery, both inertial and stable cavitation are found to play a role in enhancing drug activity and uptake. In particular, shape oscillations arising during stable cavitation are shown to provide an effective micro-pumping mechanism for enhanced mass transport across inaccessible interfaces.
\end{abstract}

Key words: High-Intensity Focussed Ultrasound (HIFU), Inertial Cavitation, Stable Cavitation, Thrombolysis, Microstreaming 


\section{INTRODUCTION}

The boundaries of biomedical acoustics are being redrawn. Having been traditionally perceived as a diagnostic modality, ultrasound is rapidly emerging as a most promising tool for non-invasive therapy and drug delivery. Low- and high-amplitude, focussed and unfocussed ultrasound fields in the frequency range $100 \mathrm{kHz}-9 \mathrm{MHz}$ propagating through tissue have been recently reported to produce a wide array of potentially beneficial bioeffects. Such phenomena include rapid and localized tissue heating (Haar \& Coussios 2007; Kennedy 2005; Leslie \& Kennedy 2007), mechanical tissue damage and homogenization (Roberts et al 2006), the dissolution of blood clots (thrombolysis) (Datta et al 2006; Everbach \& Francis 2000; Trubestein et al 1976), vascular occlusion (acoustic haemostasis) (Vaezy \& Zderic 2007), the locally enhanced and time-released activity of drugs (sonodynamic therapy) (Jeffers et al 1991; Kinoshita \& Hynynen 2006), increased and reversible permeability of cell membrane and skin to large molecules (sonoporation and sonophoresis) (Bao et al 1997; Ohl et al 2006; van Wamel et al 2006; Wu et al 1998) and the reversible opening of the blood-brain barrier (Hynynen et al 2005; McDannold et al 2006; Mesiwala et al 2002; Vykhodtseva et al 1995). In many of these therapeutic scenarios, diagnostic ultrasound provides unique opportunities for quantitative, real-time treatment monitoring, giving rise to challenges not previously encountered in traditional ultrasonography (Rivens et al 2007).

The operation transition from an imaging modality to one of therapy is quite simple: therapy tends to employ far greater average acoustic intensities than imaging. In the context of non-invasive therapies aimed at tissue destruction (ablation) or vascular 
occlusion (acoustic haemostasis), this increase is normally achieved through a combination of greater peak intensities (thousands versus tens of $\mathrm{W} / \mathrm{cm}^{2}$ ) and longer pulse durations (seconds versus microseconds). The end result is a beam of highintensity focussed ultrasound (HIFU) that can deposit energy deep within tissue onto a region about the size of a grain of rice. In that region, viscous absorption of the mechanical energy carried by the ultrasound wave into heat can lead to cell death by thermal necrosis, leaving tissue outside the HIFU focal region relatively unaffected (Bailey et al 2003; Haar \& Coussios 2007).

High-Intensity Focussed Ultrasound (HIFU) is rapidly emerging as the most promising modality for the treatment of deep-seated solid tumours, being the only form of localized ablative therapy that is truly non-invasive. However, its widespread clinical uptake is being hindered by the relatively long treatment times, the inability to monitor tissue destruction in real time and the risk of causing unwanted tissue damage ahead of the targeted region (Kennedy 2005). The acoustic absorption and attenuation coefficient in most tissues is a power law of frequency (Goss et al 1980; Goss et al 1978a) and this inevitably leads to a compromise between the rate of energy deposition and the depth at which treatment can be delivered: higher ultrasound frequencies are readily absorbed into heat at the focus but are also heavily attenuated along the ultrasound propagation path, which can lead to undesirable pre-focal damage (Haar \& Coussios 2007). Both the speed and safety of non-invasive tissue ablation by ultrasound therefore depend on the ability to achieve much higher energy deposition efficiencies at the focus compared to the pre-focal region, whilst developing techniques for the real-time monitoring of tissue destruction, 
either using ultrasound or magnetic resonance imaging (MRI), remains one of the biggest challenges in HIFU therapy (Rivens et al 2007).

In the context of drug delivery, the ability to deliver therapeutic agents directly to specific cell types or tissue regions with minimal systemic toxicity constitutes a major hurdle in current pharmaceutical development. Concentrating such agents at sites of pathology by means of molecular targeting of high-capacity therapeutic carriers (Allen \& Cullis 2004) is a fundamental initial step in such a process. Ultrasound can play a significant role in this scenario, as lipid- or protein-based vesicles encapsulating both a gas and a drug in liquid form can be activated non-invasively by ultrasound at a site of interest (Allen et al 2002; Allen \& Cullis 2004; Arora \& Ohl 2005; Frinking et al 1998; Marin et al 2001; McPherson \& Holland 2003; Munshi et al 1997; Rapoport et al 2007; van Wamel et al 2005).

The primary issue and main motivator behind targeted drug delivery protocols is systemic toxicity. A free drug can only be injected systemically at dosages well tolerated by the most sensitive organs, whilst administration of an encapsulated drug, which is generally inactive while associated with the carrier, is limited by carrier toxicity and elimination. There is therefore a huge need to identify mechanisms that can locally enhance the therapeutic activity of a pharmaceutical agent. One such example is the use of hyperthermia (40-44 C) in cancer therapy, which is believed to render tumour cells more fragile to chemotherapy, whilst leaving healthy tissue relatively unaffected (Falk \& Issels 2001; Kinoshita \& Hynynen 2006).

However, even if a therapeutically relevant dosage of the drug can be made available to the target tissue, bioavailability of the drug cannot be guaranteed. In the 
context of ischaemic stroke, the presence of a thrombus obstructing a blood vessel will lead to a severe reduction or cessation of flow, making it difficult for a drug such as recombinant tissue plasminogen activator (rt-PA) to reach the offending blood clot. The issue is further complicated by the fact that this drug relies on the plentiful supply of plasma in its vicinity in order to achieve a thrombolytic effect (Datta et al 2006; Lauer et al 1992; McPherson \& Holland 2003). Similarly, drug transport to solid tumours is severely hindered by the barrier presented by the tumour vascular endothelium, which can prevent transport across vasculature walls into surrounding tissue (Molema et al 1997). Furthermore, transport through interstitial space within a tumour occurs either via diffusion or by convection via the tumour vasculature (Jang et al 2003). However, the highly heterogenous vasculature in most solid tumours can result in high concentrations of drug in one part of a tumour but not in another, causing difficulties in treating cancer cells located distal to blood vessels.

High amplitude acoustic waves propagating through tissue can spontaneously nucleate and excite small, micron-sized bubbles, a phenomenon known as acoustic cavitation. Our hypothesis, based on our own findings and on published studies by other investigators, is that acoustic cavitation gives rise to a range of mechanical and thermal mechanisms that can enable rapid and highly localized heat deposition, enhance drug transport across inaccessible interfaces, facilitate the uptake of drug by cells, and locally enhance the therapeutic efficacy of a pharmaceutical agent. The objective of this paper is to provide a general overview of the mechanisms by which acoustic waves can enable non-invasive therapy and targeted drug delivery, the former relying entirely on the efficacious deposition of energy to permanently alter the target tissue, and the latter on 
the synergistic effect of ultrasound and a disease-specific pharmaceutical agent for maximal therapeutic effect. Then, by providing a qualitative and quantitative description of acoustic cavitation and some of its associated bioeffects, the reader will be invited to appreciate how bubble activity can help address some of the major present and future therapeutic challenges, ranging from enhanced site-specific energy transfer to providing a means for real-time treatment monitoring.

\section{ACOUSTIC CAVITATION}

\subsection{Regimes of Cavitation}

The term acoustic cavitation refers loosely to the dynamical response of a bubble driven by acoustic stress. In fluidic systems devoid of pre-existing bubbles, this process encompasses two stages: bubble nucleation followed by driven cavitation activity. The mechanisms for nucleation are quite diverse, ranging from heterogeneous nucleation on imperfectly wetted to solid surfaces to cavity formation from the absorption of nuclear particles (Bremond et al 2005; 2006; Church 2002; Crum 1982; Miller et al 2000). Although cavitation nuclei are by no means plentiful in living tissue and blood, we assume for the purposes of this discussion that said nuclei exist a priori, and focus instead on the dynamics of an acoustically small (radius $<<\lambda$ ) gas bubble in a Newtonian fluid undergoing radial oscillations - all key approximations intended to simplify the model while retaining most of the essential underlying physics. 
Much of what we consider to be acoustic cavitation activity falls into two broad phenomenological categories. So-called stable cavitation refers to the repetitive pulsation of an acoustically driven bubble about an equilibrium radius. The response of the bubble is dictated by the compressibility of the gas phase and associated physical effects include sound scattering, cavitation microstreaming (Elder 1959; Tho et al 2007), subharmonic noise generation (Eller \& Flynn 1968; Eller 1974; Leighton \& Phelps 1997; Longuet-Higgins 1990; Prosperetti 1975), heating in the viscous boundary layer surrounding the bubble (Hilgenfeldt \& Lohse 2000; Hilgenfeldt et al 2000; Prosperetti 1977), and bubble translation due to radiation stress (Crum \& Eller 1970).

If the peak negative pressure in the sound field is increased, the bubble grows to a size where it is essentially filled with vapor with a small amount of gas. The subsequent driven collapse of this vapor cavity is quite violent and is dominated by the inertia of the inrushing fluid. Supersonic wall velocities can ensue and the associated physical effects of inertial cavitation include microstreaming, fluid jetting near boundaries, and extreme thermodynamic conditions in the bubble leading to light production (sonoluminescence) and chemical reactions (sonochemistry). In addition, one observes transient, broadband acoustic emissions associated with the large bubble wall acceleration, which can be detected remotely and provide a tool for monitoring cavitation activity non-invasively. Leighton (1994) provides a comprehensive review of cavitation phenomenology and associated physical effects. 


\subsection{The Radial Dynamics of an Acoustically Driven Bubble}

To model a process as complex and nonlinear as cavitation at intensities relevant to therapeutic ultrasound, one must apply simplifying assumptions to constrain the problem in order to reproduce macroscopic results and yield predictive insights. We assume radial pulsations of non-interacting bubbles, ignoring gas diffusion and surface instabilities, and assume that evaporation and condensation occurs instantaneously relative to the time scale for bubble motion, which is typically between $100 \mathrm{nsec}$ and $1 \mu \mathrm{sec}$. We assume that a uniform Newtonian fluid surrounds the bubble with an effective viscosity that is determined semi-empirically. This last assumption, which is clearly suspect, is supported in part by the fact that tissue relaxation time scales tend to be far longer than acoustic time scales and shear thinning is not important in the limit of very high strain rates.

There are a number of radial equations of motion suitable for modeling bubbles dynamics at typical HIFU frequencies and pressure amplitudes. We employed one of the more popular formulations from Keller and Miksis (1980) :

$$
\begin{aligned}
\left(1-\frac{1}{c_{L}} \frac{d R}{d t}\right) R \frac{d^{2} R}{d t^{2}}+\frac{3}{2}\left(1-\frac{1}{3 c_{L}} \frac{d R}{d t}\right)\left(\frac{d R}{d t}\right)^{2} & =\frac{1}{\rho_{L}}\left(1+\frac{1}{c_{L}} \frac{d R}{d t}+\frac{R}{c_{L}} \frac{d}{d t}\right) p_{B} \\
& -\frac{1}{\rho_{L}}\left(1+\frac{1}{c_{L}} \frac{d R}{d t}\right)\left(p_{\infty}-p_{a}\left(t+\frac{R}{c_{L}}\right)\right)
\end{aligned}
$$

where

$$
p=p_{B}+\frac{2 \sigma}{R}+4 \mu_{L} \frac{1}{R} \frac{d R}{d t} .
$$


Here $R$ is the instantaneous bubble radius, $p$ is the internal pressure in the bubble, $P_{\infty}$ is the ambient pressure at infinity, $p_{a}(t)$ is the applied acoustic pressure, $p_{B}$ is the pressure on the wet side of the bubble wall, $c_{L}$ is the sound speed in the liquid and $\rho_{L}$ is the density of the liquid, $\sigma$ is the surface tension and $\mu_{L}$ is the dynamic viscosity. This is essentially a force balance equation applied across the bubble wall and accounts for the compressibility of the surrounding medium, which we assume uniform and bubble free.

The internal pressure of the bubble is well described by (Prosperetti et al 1988):

$$
\frac{d p}{d t}=\frac{3}{R}\left\lfloor\left.(\gamma-1) K \frac{\partial T}{\partial r}\right|_{R}-\gamma p \frac{d R}{d t}\right\rfloor,
$$

where $\gamma$ and $K$ are the ratio of specific heats and thermal conductivity of the gas, respectively. The temperature field is given by

$$
\begin{aligned}
& \frac{\gamma}{\gamma-1} \frac{p}{T}\left(\frac{\partial T}{\partial t}+v \frac{\partial T}{\partial r}\right)=\frac{d p}{d t}+\frac{1}{r^{2}} \frac{\partial}{\partial r}\left(\kappa r^{2} \frac{\partial T}{\partial r}\right) \text { and } \\
& v=\frac{1}{\gamma p}\left[(\gamma-1) K \frac{\partial T}{\partial r}-\frac{1}{3} r \frac{d p}{d t}\right] .
\end{aligned}
$$

where $r$ is the radial coordinate, $v$ is the radial velocity of the gas, and $\kappa$ is the polytropic index. At the boundary of the bubble, the temperature is set equal to that of the surrounding medium. The solution is obtained using a Galerkin spectral method 
following Kamath and Prosperetti (1989). The reader is also referred to Yang and Church for details (2006).

Figure 2a shows the computed steady-state radial response of a bubble in a Newtonian liquid driven at $1 \mathrm{MHz}$ and the somewhat modest pressure amplitude of $1 \mathrm{MPa}$. The liquid is assigned the same physical properties as our experimental tissue phantom, which is a suspension of graphite particles in agar gel designed to mimic the acoustical and thermal properties of non-perfused tissue, as described in Section 3. The bubble equilibrium radius, $R_{o}$ is permitted to vary between "super-resonant" (up to $50 \mu \mathrm{m}$ ) and sub-resonant (as low as $50 \mathrm{~nm}$ ), where the linear resonance radius $R_{r}$ is given by (Devin 1959):

$$
R_{r}=\frac{1}{2 \pi f} \sqrt{\frac{3 \gamma P_{\infty}}{\rho_{L}}\left(1+\frac{2 \sigma}{P_{o} R_{o}}\right)-\frac{2 \sigma}{\rho_{L} R_{o}}},
$$

For an air bubble in water, this radius is approximately $3.75 \mu \mathrm{m}$ at $1 \mathrm{MHz}$. Because the effective viscosity of the phantom gel is unknown, particularly at these scales, we choose to treat shear viscosity as an independent parameter that varies between $0.001 \mathrm{~Pa}$-sec (water) and $0.5 \mathrm{~Pa}-\mathrm{sec}$. The upper bound value is based on the measured acoustic attenuation coefficient in the phantom (order $5 \mathrm{~Np} / \mathrm{m} / \mathrm{MHz}$ ), where we assume attenuation loss is due solely to classical viscous absorption (Holt \& Roy 2005).

All regimes of acoustically driven radial bubble dynamics are illustrated in Fig. 1. Consider first the response of super-resonant bubbles. The cycle averaged maximum radius, $R_{m}$, increases more or less linearly with $R_{o}$, which suggests that the radial pulsation amplitude is relatively weak and only mildly dependent on $R_{o}$ and $\mu$. This 
muted response is due to the significant volume of mass loading the bubble; the effective mass loading on a linearly pulsating bubble is approximately equal to $3 \rho V_{o}$, where $\rho$ is the density of the liquid and $V_{o}=(4 / 3) \pi \rho R_{o}^{3} \quad$ (Leighton 1994). This portion of the parameter space corresponds to the stable cavitation regime. Next, note also the peaks in the response for micron-sized bubbles. Collectively, these represent the "monopole" resonance of the bubble (Longuet-Higgins 2006) along with harmonic and subharmonic resonances resulting from the nonlinear nature of Eq. (1). These resonances are dampened by viscosity and correspond to the resonance regime.

Finally, consider the very small, sub-micron bubbles, where $R_{m}$ exhibits a broad, nearlyflat plateau bounded by precipitous drops for high viscosities and for bubble sizes below a critical size known as the Blake radius, which is the smallest size a bubble can have before surface tension completely arrests acoustically induced bubble growth (Neppiras \& Noltingk 1951). This is because when very small bubbles grow, their internal pressure rapidly drops to the vapor pressure. If you ignore surface tension and viscous stress, the average pressure drop across the bubble wall, and the average velocity during the growth phase, is approximately the same for all $R_{0}$. Since the time scale for growth is approximately the same and the initial radius is so small, $R_{m}$ is, to first order, also independent of $R_{o}$. For reasons that will become apparent, this is the inertial cavitation regime.

During growth, the sound field does work on the fluid that is dissipated through viscous heating or sound radiation and stored as potential energy, which is subsequently released 
upon collapse. The collapse energetics scale with a quantity called the bubble expansion ratio, which is defied as $R_{m} / R_{o}$ and plotted as a function of pressure and viscosity in Fig. $3 b$. The dominant feature is the very large peak for very small bubbles. These bubbles are vapor cavities for much of their lifetimes. They grow explosively (isothermally) and collapse inertially (adiabatically), resulting in the aforementioned extreme temperature and pressure elevations in the gas. The collapse is accompanied by transient (broadband) acoustic emissions that result from the high fluid accelerations at the instant of collapse and rebound. Quite often, these bubbles will break up upon collapse, and this regime of behavior is termed "transient" cavitation.

\section{NON-INVASIVE THERAPY BY HIGH-INTENSITY FOCUSSED ULTRASOUND (HIFU)}

In the context of non-invasive cancer therapy, high-intensity focussed ultrasound (HIFU) is used to rapidly deposit heat onto a small region coincident with the focal volume to induce cell death by thermal necrosis. The shape of the region of thermal necrosis, or lesion, produced in tissue by high intensity focused ultrasound (HIFU) is determined to first order by the spatial distribution of acoustic intensity in the beam. Most of the heating is confined to the focal region, which, for a typical HIFU applicator operating at $1 \mathrm{MHz}$, is an oblate ellipsoid with a major diameter of approximately $2 \mathrm{~mm}$ and a length of about $1 \mathrm{~cm}$. Solid tumours, located at depths as great as $20 \mathrm{~cm}$ below the skin surface, can thus be treated non-invasively, either by laying multiple consecutive adjacent lesions or by continuously moving the HIFU focal volume in predetermined 'tracks' so as to 
span the entire tumour volume (ter Haar \& Coussios 2007). Both targeting and real-time monitoring of HIFU therapy constitute major challenges, and for this reason the production of well-defined, predictable and reproducible thermal lesions is highly desirable (Kennedy 2005). Furthermore, maximizing the heat deposition efficiency in the focal volume compared to prefocal heat deposition is essential, in order to minimize the risk of unwanted damage in the ultrasound propagation path. We proceed to provide a general description of heat deposition by ultrasound and to describe how acoustic cavitation could help achieve the desired therapeutic effects using much lower intensities than those currently employed in most clinical systems.

\subsection{Modelling Heat Deposition by Ultrasound}

Tissue heating by ultrasound involves an energy balance in which source terms associated with metabolic heating and ultrasound power deposition are balanced by sink terms associate with convective cooling (large blood vessels), perfusion cooling (microvasculature), heat conduction, and energy storage, with the latter leading to a temperate rise. This process is well described by the Pennes bioheat transport equation (BHTE) (Pennes 1948):

$$
\begin{aligned}
& \rho_{t} C_{t} \frac{\partial T}{\partial t}=K_{t} \nabla^{2} T-w_{b} C_{b}\left(T-T_{\infty}\right)+q_{s} \\
& \rho_{b} C_{b} \frac{\partial T}{\partial t}=K_{b} \nabla^{2} T-\rho_{b} C_{b}(\vec{u} \cdot \vec{\nabla} T)+q_{s} \\
& \vec{u}=\vec{u}_{e x t}+\vec{u}_{s t r}=2 U_{0}\left[1-\left(\frac{r}{r_{0}}\right)^{2}\right]+\vec{u}_{s t r}
\end{aligned}
$$


where $T(r, t)$ the temperature and $\rho, C$, and $K$ are the density, specific heat, and thermal conductivity of the medium, which is taken as either tissue (subscript " $t$ ") or flowing blood (subscript " $b$ "). The contribution of ultrasound heating appears as a source term, $q_{s}$, which is the power deposited per unit volume of tissue. For a non-relaxing medium, this is given by (Pierce 1981):

$$
q_{s}=2 \alpha I=\frac{2 \alpha}{\omega^{2} \rho c}\left\langle\left(\frac{\partial p}{\partial t}\right)^{2}\right\rangle,
$$

where $I$ is the local acoustic intensity, $p$ the local acoustic pressure, $\alpha$ is the local acoustic absorption coefficient, and the time average is carried out over one acoustic cycle.

Note that there are separate BHTE equations for the tissue and blood domains. In the former, $w_{b}$ is the perfusion rate, which represents the average mass flow of blood in the capillary bed. In the latter, the BHTE includes a convective transport term in which $T_{\infty}$ is the temperature far away from the HIFU focus and $\vec{u}$ is the blood flow velocity field. This flow field, which varies with the local conditions, is expressed in Eq (5) as the linear sum of a fully developed parabolic flow in a pipe of radius $r_{o}$ and an acoustic streaming flow. Note that the average externally-driven flow velocity, $U_{o}$, is pulsatile in that it fluctuates with the cardiac cycle.

There is a considerably body of literature devoted to acoustic streaming, which is in essence a transfer of momentum between sound waves and quasi-steady fluid motion 
(Nyborg 1965). With the possible exception of smaller vessels, acoustic streaming velocities are typically less than external flow velocities. Moreover, although it is possible to heat flowing blood, the primary contribution of large vessels is to convectively cool surrounding tissue (Huang et al 2004). In this sense, the presence of vessels within the treatment volume can be quite problematic, and involves modeling aspects that lie beyond the scope of this discussion. Therefore, in the pages to follow, we consider non-perfused, physically uniform, Newtonian viscous media.

Figure 2a shows the peak temperature elevation measured in a tissue mimicking "phantom" consisting of a suspension of graphite particles in an agar gel (Holt \& Roy 2001; 2005). Measurements were obtained using a fine wire thermocouple positioned in the focal plane and $0.5 \mathrm{~mm}$ off the axis of the HIFU source. The solid line was computed using a finite difference time domain simulation of Eq. (1), where the pressure field was computed using the Westevelt equation for nonlinear sound propagation (Pierce 1981) and the pressure and temperature equations were coupled using Eq. (2). Calculations employed independently measured phantom material properties and no parameter fitting was employed.

Consider first the data obtained for pressures less than 1.1 MPa; note the excellent agreement between model and measurement. For the relatively modest HIFU pressure employed, the temperature elevation is quadratic in the peak pressure amplitude and thus linear in the acoustic intensity. For the purpose of this discussion, we call this the "linear heating" regime. 
If significantly higher pressure amplitudes are employed, the initially monochromatic waves can become increasingly nonlinear, for at finite amplitudes the sound speed is a function of the local pressure (ter Haar \& Coussios 2007; Khokhlova et al 2006). This results in the formation of harmonics in the beam, as energy is converted from the low to high frequencies. Absorption in tissue is frequency dependent, and the following empirical model has been shown to work well for a large range of tissues:

$$
\alpha(f)=\alpha_{o}+\alpha_{1} f^{1.1} \quad[\mathrm{~dB} / \mathrm{cm}],
$$

where the coefficients depend on the specific tissue type (Goss et al 1980; Goss et al 1978b). The harmonic components of the nonlinear beam are more readily absorbed and the net result is enhanced energy deposition. Khokhlova et al. have studied this extensively (2006) and shown that, at very high HIFU intensities - of order $5000 \mathrm{~W} / \mathrm{cm}^{2}$ - nonlinear heating can be the dominant mechanisms, with calculations suggesting that near-boiling temperatures can be attained in less than a second of exposure. The appearance of boiling bubbles in the focal region can be highly undesirable, as increased scattering by vapour cavities can help promote near-field heating and lead to the production of 'tadpole' lesions that are unpredictable in shape, appear bulbous at the proximal end and grow uncontrollably towards the transducer rather than symmetrically relative to the focal plane (Chen et al 2003; Khokhlova et al 2006). The formation of lesions that are unpredictable in size and position is one of the principal technology barriers to safe and effective HIFU surgery. 


\subsection{Cavitation-Enhanced Heating From HIFU}

Consider now HIFU heating at pressure amplitudes that are slightly higher than those required to generate predominantly linear heating. Figure $2 \mathrm{~b}$ depicts the heating and cooling curves measured in our agar-graphite phantom. The bottom five curves were obtained for pressure amplitudes ranging from $0.6 \mathrm{MPa}$ to $1.1 \mathrm{MPa}$ and correspond to the linear heating regime in Fig. 2a. Note, however, the large increase in temperature rise induced when one goes from 1.1 $\mathrm{MPa}$ to $1.2 \mathrm{MPa}$ a 9\% increase in pressure incurrs a $330 \%$ increase in temperature elevation. A large change in themo-fluidic response (temperature, flow, noise, etc.) brought on by a small change in driving pressure is a classic indicator of the onset of acoustic cavitation activity. The critical pressure, called the cavitation threshold pressure, represents either a threshold for cavitation nucleation or for the transition from stable to inertial bubble response. In most cases involving HIFU in tissues, nucleation is the most important precursor to cavitation production.

This progression from linear heating to cavitation enhanced heating is further portrayed in Fig. 2a, where we extend the range to include data obtained at super-threshold pressures. Beyond the enhancement region, the temperature rise appears to saturate, and even diminishes as one increases the pressure further. This is likely caused by an excess of bubble activity in the focal region, in which case the thermocouple is shielded from the HIFU beam by sound scattering off the perimeter of the bubbly region. Note also the increased uncertainty in the measurements obtained at and above the threshold pressure, 
another indicator of cavitation activity, which can be sporadic in nature. The literature reports several examples of cavitation enhanced HIFU heating in tissue phantoms as well as tissues ex vivo and in vivo (Coussios et al 2007).

\subsection{Modeling Cavitation-Enhanced Heating}

The pathway for accelerated heating from cavitation is the process by which bubbles convert acoustical energy into mechanical energy, as described by Eqs. 3-5, which is subsequently dissipated as heat. Although several mechanisms have been proposed in the

literature, two stand out as the most efficient way to effect said transfer given purely radial bubble motion (Coussios et al 2007; Devin 1959; Hilgenfeldt \& Lohse 2000; Hilgenfeldt et al 2000; Holt \& Roy 2005; Prosperetti 1977): viscous heating in the boundary layer of larger, stable cavitation bubbles and the absorption of broad-band acoustic emissions from inertial cavitation. To estimate the cycle-averaged power dissipated through viscous heating at the bubble wall, we multiply the viscous force at the surface times the wall velocity

$$
W_{\text {vis }}=\langle\vec{F} \cdot \dot{R}\rangle_{t}=\left\langle\left(\frac{4 \mu_{L} \dot{R}}{R}\right)\left(4 \pi R^{2}\right) \cdot \dot{R}\right\rangle_{t}=16 \pi \mu_{L}\left\langle R \dot{R}^{2}\right\rangle_{t}
$$

where the average is evaluated over one acoustic cycle and $R(t)$ is computed from the bubble dynamics model presented earlier.

A bubble undergoing radial pulsations emits like a volume oscillator: 


$$
p_{r a d}(r, t)=\frac{\rho_{L}}{4 \pi r} \frac{d^{2} V}{d t^{2}} e^{-\alpha(r-R)}=\frac{\rho_{L} R}{r}\left\lfloor 2\left(\frac{d R}{d t}\right)^{2}+r \frac{d^{2} R}{d t^{2}}\right\rfloor e^{-\alpha(r-R)},
$$

where $p_{\text {rad }}$ is the radiated pressure and $V$ is the instantaneous volume of the bubble and the absorption coefficient is, in general, frequency dependent. From this one can evaluate the average power radiated by the bubble:

$$
W_{\text {rad }}(r)=4 \pi r^{2} I=\frac{4 \pi r^{2}}{\rho_{L} C_{L}} \int_{0}^{\infty} p_{r a d}^{2}(r, t) d t=\frac{4 \pi r^{2}}{\rho_{L} C_{L}} \int_{-\infty}^{\infty} \hat{p}_{r a d}^{2}(r, f) d f \quad .
$$

Following Hilgenfeldt et al. (Hilgenfeldt \& Lohse 2000; 2000), we express the power in terms of the Fourier transform of the pressure, as this supports the use of a frequency dependent absorption coefficient. Evaluating Eqs. (8) and (9) yields the power radiated by the source. The power deposited by the absorption of acoustic emissions into the tissue within a spherical volume bounded by $\mathrm{r}$ is thus:

$$
W_{a c}(r)=W_{o}-W_{r a d}(r)
$$

Equations (7) and (8) yield the power deposited into the medium by radial bubble motion. To incorporate them into the BHTE, Eq. (1), we define the source terms for bubble enhanced heating, expressed as power densities: 


$$
W_{v i s}=\int_{r=R}^{\infty} q_{v i s} d V \quad ; \quad W_{a c}=\int_{r=R}^{\infty} q_{a c} d V
$$

The BHTE applied to the tissue domain takes the form:

$$
\rho_{t} C_{t} \frac{\partial T}{\partial t}=K_{t} \nabla^{2} T-w_{b} C_{b}\left(T-T_{\infty}\right)+\frac{2 \alpha}{\omega^{2} \rho c}\left\langle\left(\frac{\partial p}{\partial t}\right)^{2}\right\rangle+q_{v i s}+q_{a c}
$$

Figure 3 shows the calculated thermal power deposited per bubble when driven by a 1 $\mathrm{MHz}$ HIFU field with a pressure amplitude of 2.0 MPa over roughly the same parameter space considered in Fig. 1. Power deposited by absorbed acoustic emissions is on the order of 25-30 $\mathrm{mW}$ for bubbles smaller than resonance size and viscosities less then 10 time that of water. Alternatively, viscous boundary layer heating results in similar levels of power deposition, but only for super resonant bubbles at the very highest viscosities. Calculations indicate that the number of bubbles required to account for the experimentally observed levels of enhanced heating is on the order of 10 for both heating mechanisms. Clearly, we have two viable models for bubble enhanced heating, but they cannot simultaneously apply to the same bubble population and in the same medium.

\subsection{Cavitation Monitoring and Noise Diagnostics}

One physical observable that clearly delineates stable from inertial cavitation is noise production. Indeed, the latter results in broadband acoustic emissions that are the very source of the heating effect. By using a passive acoustic sensor, tuned to the higher 
frequencies, one can non-invasively monitor cavitation noise emanating from the HIFU focus. The use of passive cavitation detection (PCD) to detect and characterize cavitation activity is well established (ANSI2002; Leighton 1997). We employed a focused sensor, confocally positioned with the HIFU transducer, and high pass filtered above $2 \mathrm{MHz}$ to isolate inertial cavitation noise from scattering of the primary HIFU field by stable cavitation bubbles. Figure 4 shows three heating/cooling curves, obtained form a similar agar-graphite phantom used to generate Fig. 3. Also plotted is the peak detected noise amplitude of the noise emanating from the confocal zone; see (Coussios et al 2007; Holt \& Roy 2001; 2005) for details on the experimental arrangement. Note that on Fig. 3b, the onset of noise production is well demarcated and correlates with an immediate increase in heating rate. This supports the contention that inertial cavitation is thermally relevant, but does not rule out a similar role for stable cavitation.

Nonetheless, the fact that noise and heating are correlated is very significant, for it suggests the possibility that, by monitoring noise, one can sense the onset and extent of bubble-enhanced heating. This approach is being pursued by several groups (Coussios et al 2006; Coussios et al 2007; Rabkin et al 2005; Rivens et al 2007; Thomas et al 2005), and may eventually serve as the basis for a sensing strategy for guiding bubble-enhanced HIFU in the clinic. 


\subsection{Allowable Bubble Sizes in HIFU}

When a bubble is nucleated by HIFU, once of several things can happen, depending on the bubble size, the pressure amplitude, the dissolved gas content in the liquid or tissue, and the viscosity. The bubble can dissolve under the effect of surface tension and disappear, or undergo inertial cavitation and break up on collapse. A pulsating bubble can also be made to grow over several acoustic cycles due to an asymmetry in the concentration gradient / surface area relationship that causes more gas to diffuse into an expanded bubble than is forced out of a compressed bubble, a phenomenon known as rectified diffusion (Eller \& Flynn 1963). In this regime, the bubble can grow until it reaches a size where mass loading inhibits radial pulsations to the point where rectification stops and the bubble retains a stable equilibrium size (the rectified diffusion threshold size). As this equilibrium size increases to a critical value, a parametric instability to wavy shape perturbations can lead to the excitation of shape oscillations (Crum \& Eller 1970; Eller \& Crum 1970; Maksimov \& Leighton 2001). These can reach amplitudes large enough to cause the bubble to fragment into smaller pieces that can once more respond inertially or dissolve due to surface tension.

The lower bound for "allowable" bubble sizes is the Blake radius, which increases with increasing pressure amplitude (Neppiras \& Noltingk 1951). The upper bound is set by rectified diffusion for higher viscosity media or shape instabilities for lower viscosity media. As a general rule of thumb, HIFU-induced cavitation fields have bubbles that are 
bound by the Blake Radius (order $100 \mathrm{~nm}$ ) at the lower end and resonance size (on the order of $4 \mu \mathrm{m}$ at $1 \mathrm{MHz}$ ) at the upper end.

Yang et al. (2004) investigated the role of surface instabilities in HIFU. For resonance and sub-resonant bubbles, they assumed that shape distortions could be modeled as the superposition of discrete modal surface oscillations with amplitude $a_{n}(t)$. Using a perturbation analysis, the modes that possessed amplitudes that grew over time were identified. The criterion for bubble breakup was $\left|a_{n}(t)\right| \geq \mathrm{R}(\mathrm{t})$, and the model accounted for both Rayleigh-Taylor and parametric instability. For larger bubbles, Yang et al. assumed that the Faraday instability dominated. They then assumed that the frequency of the most unstable mode is exactly $1 / 2$ the driving frequency and determining the critical value of the viscosity below which the breakup criterion is satisfied for a given acoustic pressure amplitude.

By combining rectified diffusion and shape instability calculations one can determine stability boundaries over a specified range of bubble sizes and viscosities. It is found that the large, very high viscosity bubbles that account for maximal viscous boundary layer heating were in fact excluded because the requisite tissue viscosity is so large that it is not possible to get bubbles to grow to that size via rectified diffusion. Indeed, the calculations of Yang et al. (2004) support the hypothesis that, unless the large superresonant bubbles are introduced into the medium by some other means, inertial cavitation, and only inertial cavitation, is the cause of bubble-enhanced heating. This 
observation is consistent with results reported by Hilgenfeldt et al. (Hilgenfeldt \& Lohse 2000; 2000) and Lafon et al. (2005).

\subsection{Challenges of Cavitation-Enhanced Heating: Nucleation and Control}

Inertial cavitation shows tremendous potential as a means of dramatically enhancing heat deposition at the focus during HIFU exposure, making it possible to achieve the desired therapeutic effect by utilizing much lower intensities than those employed in most clinical systems. This increased heat deposition efficiency arises from the conversion of part of the energy carried by the incident wave into broadband noise emissions, which are readily and very locally absorbed at the HIFU focus. As illustrated in Fig. 4 for a cavitation-promoting agar-graphite tissue mimicking material, a modest increase in insonation pressure beyond the cavitation threshold results in a six-fold increase in the local rate of heating. Futhermore, monitoring of the broadband noise emissions associated with inertial cavitation could provide an inexpensive means of monitoring HIFU therapy in real time.

In order to avoid undesirable prefocal damage and the formation of poorly controlled tadpole-shaped lesions, cavitation enhancement of HIFU therapy is best achieved using low-to-moderate HIFU intensities, typically on the order of a few hundreds of W/cm². The use of low intensities is also desirable because cavitation-enhanced heating is most effective at tissue temperatures well below boiling, due to the fact that elevated vapor pressures serve to mitigate violent collapse. In the boiling cavitation regime, the bubbles 
can become quite large as they fill with vapor, and the dominant mechanism for enhanced heating becomes viscous boundary layer heating.

However, in order to achieve cavitation-enhanced heating at low intensities, one must first exceed the nucleation threshold pressure, which can be as high as $10 \mathrm{MPa}$ (on the order of $3 \mathrm{~kW} / \mathrm{cm}^{2}$ ) in some tissues (Church 2002; Tran et al 2003; Xu et al 2005). Several techniques have been proposed for promoting cavitation nucleation both in vivo and ex vivo. A number of independent studies have shown that very short, large amplitude "conditioning pulses" followed by lower amplitude continuous-wave focussed ultrasound exposure can both initiate and sustain controlled levels of cavitation activity (Parsons et al 2006; Sokka et al 2005; Sokka et al 2003; Xu et al 2006). Systemically applied ultrasound contrast agents can serve as effective nucleation sites in the blood phase (Miller \& Thomas 1995; Tran et al 2003; Tu et al 2006a; Tu et al 2006b). Investigators have even used the combination of pulsed laser light and gold nanoparticles to produce cavitation nuclei "on demand" (Farny et al 2005). Nevertheless, the authors view the nucleation problem as a key technology barrier to the realization of bubble assisted HIFU in the clinic, as bubble nucleation is a necessary precursor to cavitation activity.

Finally, it is worth noting that, when a bubble forms at the HIFU focus and eventually breaks up, either from inertial collapse or shape instability, the medium is "seeded" with a large number of additional cavitation nuclei which may spawn cavitation in their own right and create a bubble cloud. If the pressure in the pre-focal region exceeds the 
cavitation threshold, this will provide the impetus for the bubble cloud to grow unstably towards the HIFU transducer, shielding the original focus and leading to cavitationenhanced heating pre-focally. In order to ensure the safe and effective implementation of cavitation-enhanced heating, it is therefore essential to develop protocols that make it possible to sustain and confine cavitation activity to the focal region. A number of such techniques have been proposed, which include the use of pulsed excitation to enable the dissolution of pre-formed nuclei during the quiescent period (Coussios et al 2006; Thomas et al 2005) and the use of dual-frequency methods (Sokka et al 2005). However, much work remains to be done in this area. Ultimately, the development of improved cavitation control protocols will necessitate an improved understanding of cavitation dynamics in temperature-varying viscoelastic media ((Yang \& Church 2005)

\section{ULTRASOUND ENHANCEMENT OF DRUG ACTIVITY}

The considerable potential of acoustic waves to locally release intravenously injected vesicles containing both a drug and a gas by activating the encapsulated gas bubble in order to destroy the stabilizing lipid or protein shell has been extensively reviewed elsewhere (Allen et al 2002; Borden et al 2005; Marmottant et al 2005; Stride \& Saffari 2003; Tartis et al 2006) and will not be repeated here. Instead, we focus on the mechanisms by which ultrasound can enhance the efficacy of a drug that is already present at a site of pathology. These mechanisms are of particular interest in conjunction with pharmaceutical agents that yield detrimental side-effects when injected systemically, such as chemotherapy agents in the context of cancer therapy or thrombolytic drugs in the 
context of thrombosis and stroke, whereby it is desirable to achieve a satisfactory therapeutic outcome using minimal drug dosages.

\subsection{Inertial Cavitation Mechanisms}

In the context of drug delivery, the role of inertial cavitation has been explored almost exclusively in cell suspensions in vitro. Exposure of cell suspensions to inertial cavitation in the presence of a green-fluorescent molecule (calcein) has shown that (i) cells that are proximal to the inertially cavitating microbubbles are mechanically destroyed, (ii) those at mid-distances from the cavitating microbubbles exhibit reversible poration and an increased uptake of calcein, a phenomenon known as sonoporation and (iii) those cells far away from the bubbles remain unaffected (Guzman et al 2003; Hallow et al 2006; Ohl et al 2006; Prentice et al 2005; Schlicher et al 2006).

Even though the strong correlation demonstrated by Hallow et al (2006) between the levels of broadband noise activity and sonoporation leaves little doubt as to the fact that inertial cavitation is to blame, the exact mechanism by which inertially cavitating microbubbles alter cell porosity remains poorly understood. It has been speculated that the proximity of a bubble to a cellular interface causes it to collapse asymmetrically and that its involution results in the formation of a micro-jet that serves to porate the cell membrane. However, recent, high-frame-rate optical investigations (Garbin et al 2007) have shown that micro-jetting tends not to occur for bubbles proximal to a compliant 
interface, such as a cell layer that is not in close proximity to a solid substrate such as a Petri dish.

It must also be noted that in most of the aforementioned studies, inertial cavitation has been promoted by the introduction of stabilized gas bubbles (ultrasound contrast agents) into the cell suspension, with relative disregard for what the corresponding nucleation environment would be in a physiological context. For example, there are at present few studies of any differences in nucleation threshold between healthy vasculature and that of more complex pathological structures, such as tumours (Rapoport et al 2007).

As much as in vitro studies serve to illustrate the two main types of biomechanical effects induced by inertial cavitation, namely cell death and a reversible increase in cell membrane permeability, considerable additional research is needed to ensure adequate nucleation, control and monitoring of cavitation activity in vivo. Nonetheless, the strong correlation between the level of cavitation activity and the resulting level of sonoporation suggests a noninvasive feedback method to control the bioeffects of ultrasound in real time. Both the benefits and challenges ahead are in many ways similar to those encountered in cavitation-enhanced HIFU.

\subsection{Stable cavitation mechanisms}

The relevance of stable cavitation to drug delivery has been explored almost exclusively in the context of thrombolysis, whereby a thrombolytic drug, recombinant tissue plasminogen activator (rt-PA) is administered to dissolve a blood clot obstructing a 
vessel. The drug combines with plasminogen, which is naturally dissolved in blood plasma, to form plasmin, which in turn acts to dissolve the fibrin mesh that stabilizes the blood clot (a process known as fibrinolysis). It is worth noting that the efficacy of the drug depends on having both sufficient rt-PA and sufficient plasminogen in the clot vicinity. Use of increased dosages of rt-PA poses a considerable risk of intracerebral hemorrhage, and would therefore be desirable to achieve the desired thrombolytic effect under the influence of ultrasound whilst eliminating the risk of hemorrhage outside the sound field.

Ultrasound has been shown to increase the enzymatic rate of thrombolytic therapy in vitro, in animal models and in clinical trials and this enhancement has been shown to be most pronounced at low- to mid-kHz frequencies rather than $\mathrm{MHz}$ frequencies (Datta et al 2006). This strongly suggests that ultrasound enhancement of thrombolysis is not due to thermal but rather to cavitational mechanisms (Everbach \& Francis 2000).

In a recent study, Datta et al. (2006) sought to identify the most relevant type of cavitation activity for ultrasound enhancement of thrombolysis. Porcine blood clots were immersed in porcine plasma inside a latex chamber, and exposed to combinations of rtPA and/or pulsed $120 \mathrm{kHz}$ ultrasound at an $80 \%$ duty cycle for 30 minutes in a temperature-controlled water bath. Ultrasound exposure took place using one of three amplitudes (0.15 MPa, 0.24 MPa and 0.36 MPa), the lowest having been chosen to cause no cavitation activity in plasma, the intermediate causing stable cavitation activity only and the highest as causing both stable and inertial cavitation activity. In this context, 'stable' cavitation was taken to signify prolonged non-linear bubble pulsations that give rise to emissions at subharmonics of the main excitation frequency, whilst inertial 
cavitation gave rise to broadband noise emissions, as detected by a passive cavitation detector. Thrombolytic efficacy was determined by weighing the clot before and after exposure to determine the percent mass loss over the course of the treatment, and results are summarized in Fig. 5.

A small amount of mass loss took place in the absence of ultrasound or rt-PA due to handling of the blood clot in and out of the test chamber, whilst exposure of the clot to ultrasound alone had little effect. Exposure to rt-PA only caused on average a 12\% mass loss, but addition of ultrasound in the absence of cavitation was shown to provide no benefit. By contrast, increasing the ultrasound exposure amplitude to beyond the stable cavitation threshold more than doubled the thrombolytic efficacy, leading to a $25 \%$ mass loss. Interestingly, increasing the pressure amplitude further to beyond the inertial cavitation threshold caused a reduction in thrombolytic efficacy, compared to the stable cavitation regime.

It is concluded that thrombolysis using recombinant tissue plasminogen activator is most effective when ultrasound exposure gives rise to subharmonic emissions, an observation that was recently confirmed by another investigation (Prokop et al 2007). A variety of stable cavitation phenomena can give rise to such subharmonic emissions, including shape oscillations due to surface waves and axisymmetric pulsations of bubbles with an equilibrium radius that is an integer multiple of the resonance size (Leighton 1994). Given the already large resonance size at $120 \mathrm{kHz}$, larger bubbles are unlikely to be able to exist without breaking up and it is therefore concluded that shape oscillations are responsible for the observed ultrasound enhancement of thrombolysis. 
It is speculated that the small-scale streaming flow, known as microstreaming (Elder 1959), that is generated within the oscillatory boundary layer surrounding the bubble, provides a mechanism for enhanced transport of plasma and rt-PA across the clot surface. Liu et al. (2002) have already demonstrated the effectiveness of cavitation microstreaming as a mechanism for mixing fluids at the microscale, whilst Tho et al. (2007) recently provided experimental measurements, which show that the streaming velocities of shape mode oscillating bubbles appear to be roughly 2-3 times higher than those found in volume and translating modes of oscillation. This result appears to agree with earlier observations made by Watson, Birkin \& Leighton (2003) who observed enhanced mass transfer in the vicinity of bubbles undergoing volume oscillations and also bubbles with surface waves.

The hypothesis of the shape-oscillating bubble acting as a micro-pump on the clot surface is well-supported by immuno-histochemical analysis of the thrombolyzed clots, also shown in Fig. 3. Blood clots were labeled with mouse anti-fibrinogen antibody and stained with horseradish peroxidase-linked goat anti-mouse IgG (Datta et al 2006). The untreated clot (blue) demonstrates a relatively densely staining band along the outermost surface, which is indicative of a dense fibrin mesh, whist the clot treated with ultrasound alone (green) is essentially no different. Clots treated with the thrombolytic drug rt-PA (yellow) exhibit a thinner band, whilst those treated also with ultrasound in the presence of stable cavitation (red) have a surface that appears markedly thinner, irregular and porous, which is compatible with increased bubble and microstreaming at the clot surface. 


\subsection{Future challenges: monitoring and enhancing drug activity}

In summary, what is currently known is that inertial cavitation can cause highly efficient thermal deposition, as well as direct mechanical effects resulting in cell death and increases in cell membrane permeability, whilst stable cavitation, and bubbles undergoing shape oscillations in particular, generate considerable microstreaming that leads to enhanced mixing and increased mass diffusion across interfaces. What is presently missing is an adequate understanding of how these effects translate into a physiologically relevant environment, taking account of the local cavitation nucleation environment, flow conditions, presence of blood vessels, heat advected due to blood flow and cell-bubble interactions. If the 'right' type or types of cavitation can be identified, initiated, optimized and controlled, then combinations of the aforementioned bioeffects can greatly aid targeted drug delivery for applications where the drug being administered has significant detrimental side effects and the desired delivery site is inaccessible. Such major applications include chemotherapy delivery for cancer treatment and thrombolytic delivery for stroke therapy, both of which would greatly benefit from the ability to lower systemic drug dosages for the same local therapeutic effect. Furthermore, use of cavitation to enhance therapy provides unique opportunities for non-invasive treatment monitoring, as both inertial and stable cavitation activity are accompanied by acoustic emissions that can be readily detected remotely. 


\section{ACKNOWLEDGEMENTS}

The authors would like to acknowledge the efforts of past and present students and colleagues whose work is featured in this article: R. Glynn Holt, Christy K Holland, Patrick Edson, Charles Thomas, Jinlan Huang, Xinmai Yang, Caleb Farny, Tianming Wu, Charles Thomas and Saurabh Datta. Ronald Roy's work in this area has been supported by grants from the United States Army (award number DAMD17-02-2-0014, for which The U.S. Army Medical Research Acquisition Activity, 820 Chandler Street, Fort Detrick, MD is the awarding and administering acquisition office), and the Center for Subsurface Sensing and Imaging Systems (funded under the Engineering Research Centers Program of the National Science Foundation; award number EEC-9986821). Constantin Coussios' work in this area has been in part supported by the F.V. Hunt PostDoctoral Fellowship of the Acoustical Society of America and by the generous ongoing support of the UK's Engineering and Physical Sciences Research Council. 

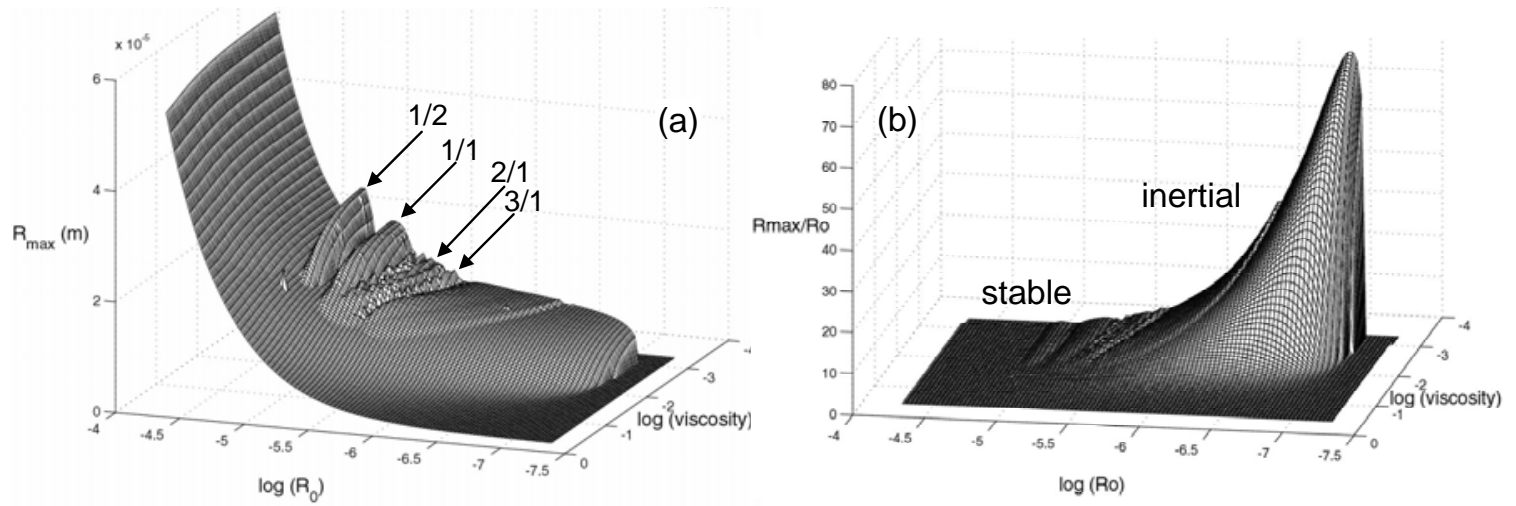

Figure 1. The cycle-averaged maximum radius (a) and expansion ratio (b) for a bubble driven by a $1 \mathrm{MHz}$ HIFU field with a pressure amplitude of $1 \mathrm{MPa}$. The acoustical and thermal properties of the surrounding medium are the same as those of the agar graphite tissue-mimicking material (Holt \& Roy 2005). 

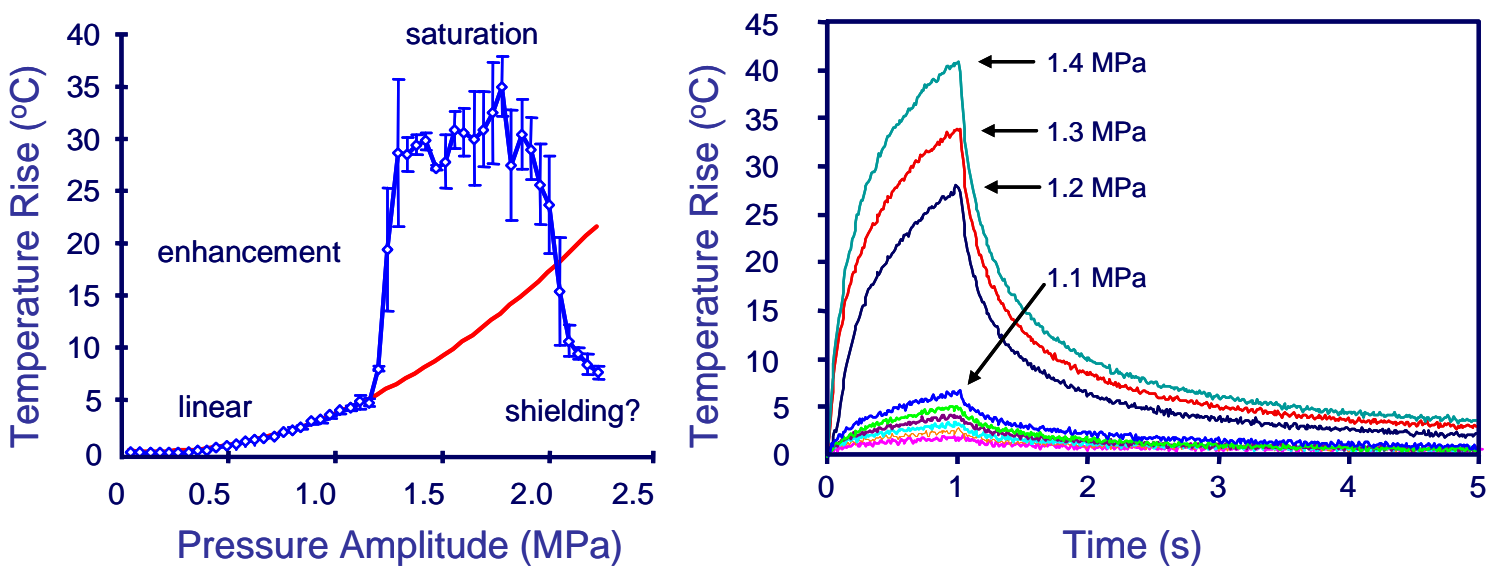

Figure 2. Temperature rise measured by a thermocouple (TC) embedded in an agargraphite tissue mimicking material exposed to $1 \mathrm{MHz} \mathrm{HIFU}$ for 1 second. A correction factor was applied that account for the so called "thermocouple artifact", which refers to enhanced heating in the viscous boundary layer surrounding the TC. (a) Measured (blue) and predicted (red) peak temperature rise versus the acoustic peak-rarefaction pressure amplitude. The error bars depict the standard deviation of 5 measurements. Measured temperature versus time for increasing peak-rarefaction pressure amplitudes; each curve corresponds to an increment of approximately $0.1 \mathrm{MPa}$ (adapted from Coussios et al 2007). 

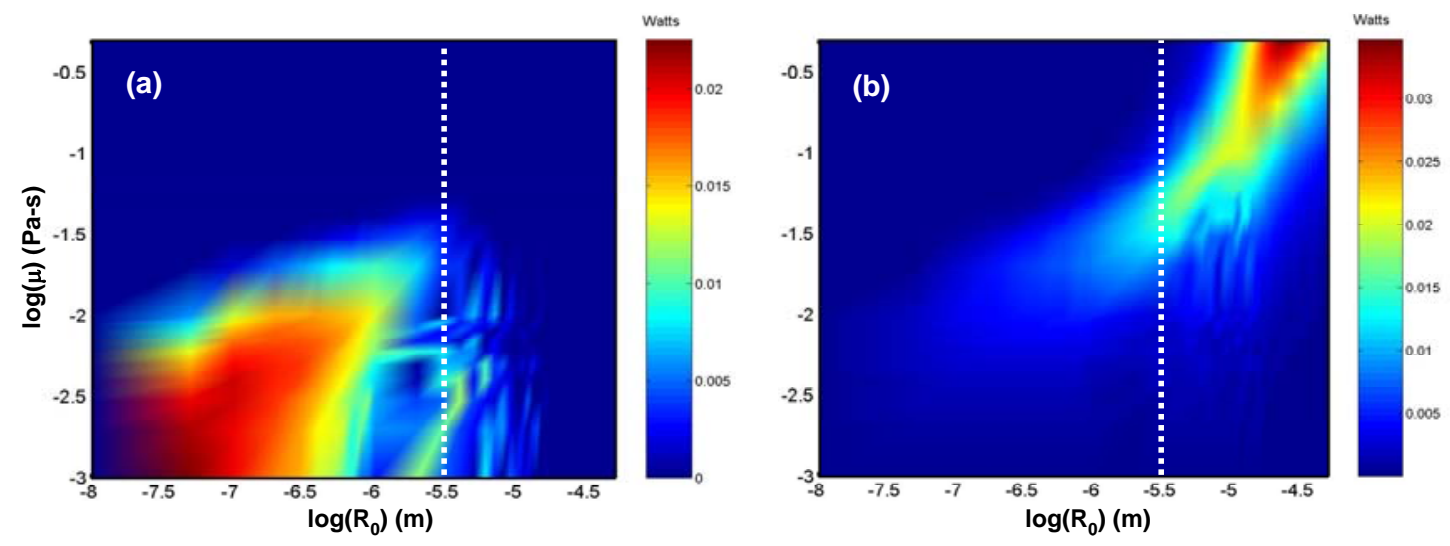

Figure 3. Thermal power deposited per bubble due to (a) broadband acoustic emissions (inertial cavitation) and (b) viscous boundary layer heating (stable cavitation). The dashed white lines depict the resonance radius at the HIFU exposure frequency of 1 MHz. The pressure amplitude was 2.0 MPa. (adapted fromHolt \& Roy 2005) 


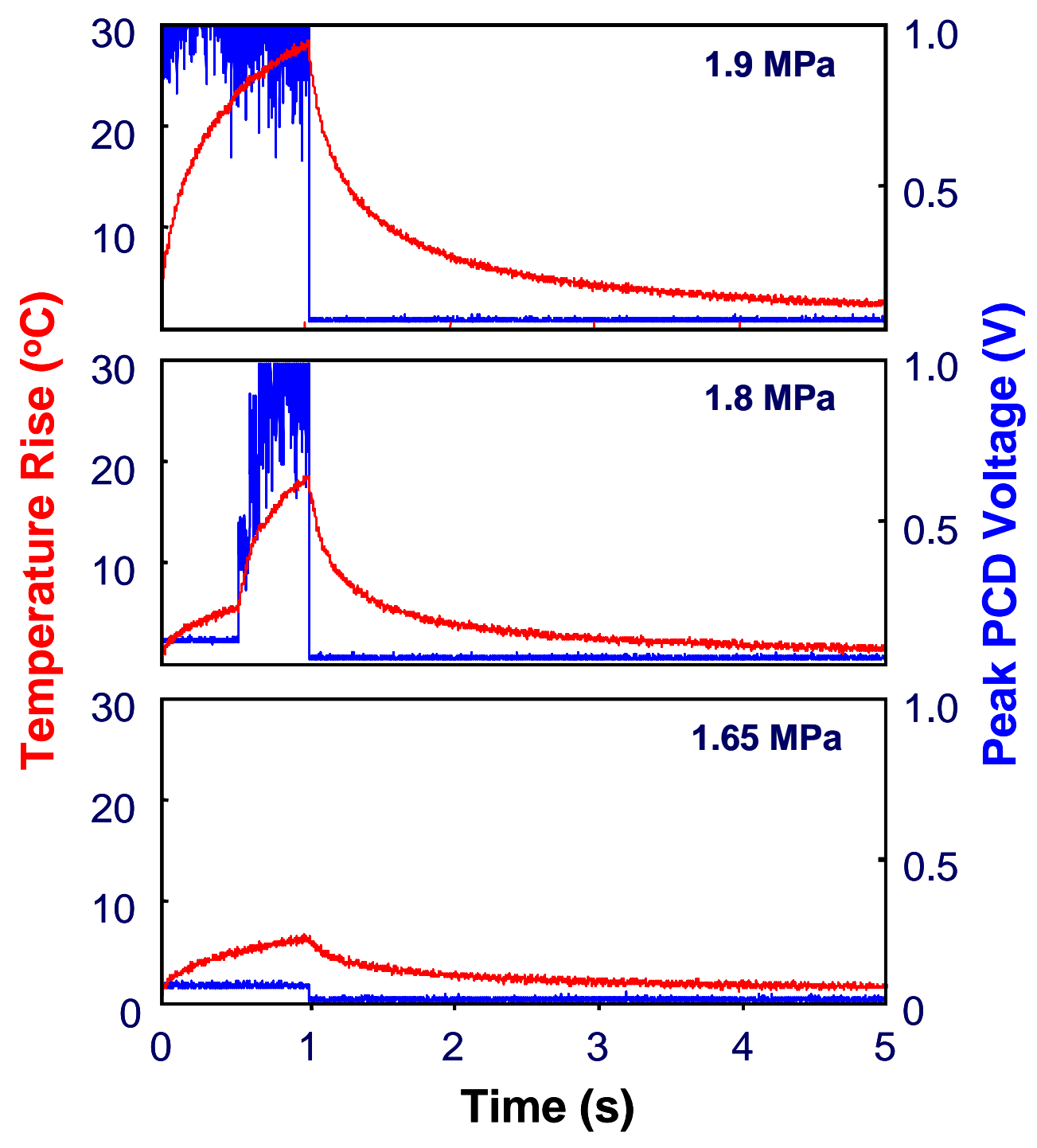

Figure 4. Temperature (solid line) and broad-band acoustic emissions (dashed line), measured with a PCD, for 1 second exposures of $1 \mathrm{MHz}$ ultrasound in the agar-graphite phantom. The nucleation threshold pressure (1.8 MPa) is greater than in Fig. 1 because this particular phantom was more thoroughly degassed. The pedestal in the PCD data in plot (c) is due to scattering of the HIFU harmonics into the detector aperture. It is not indicative of inertial cavitation activity.(adapted fromHolt \& Roy 2005) 


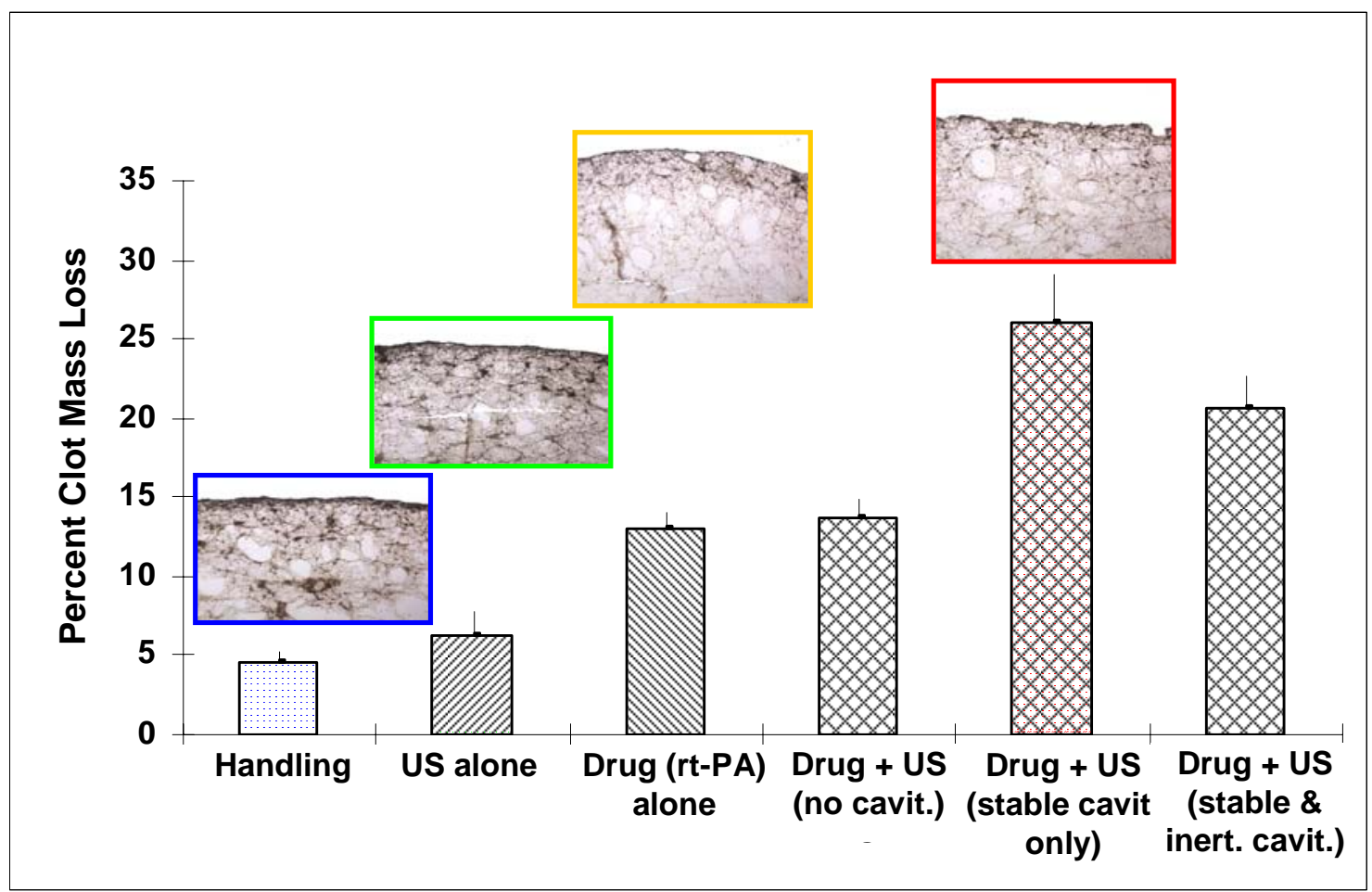

Figure 5: Percent clot mass loss following 30 minute treatment of porcine blood clots. $\mathrm{N}=5$ for each treatment protocol and error bars indicate one standard deviation. "Handling" refers to clots in plasma alone (without the thrombolyitic drug rt-PA or pulsed ultrasound). "US alone" refers to clots without rt-PA, but exposed to pulsed 120 $\mathrm{kHz}$ ultrasound at an $80 \%$ duty cycle and at $0.36 \mathrm{MPa}$ peak rarefaction pressure, "Sham" refers to clots exposed to rt-PA alone, but without pulsed ultrasound. "No cavit." refers to clots exposed to rt-PA and pulsed ultrasound below both the stable cavitation and inertial cavitation thresholds at a peak rarefaction pressure of $0.15 \mathrm{MPa}$. "Stable cavit." refers to clots with rt-PA and pulsed ultrasound above the stable but below the inertial cavitation threshold at a $0.24 \mathrm{MPa}$ peak rarefaction pressure, "Stable and Inert. cavit." refers to clots exposed to rt-PA and pulsed ultrasound above the stable and inertial cavitation thresholds at $0.36 \mathrm{MPa}$ peak rarefaction pressure. Inset photographs show immunohistochemical imaging analysis of treated clots. (adapted from Datta et al 2006) 


\section{REFERENCES}

2002. ANSI technical report: bubble detection and cavitation monitoring, Standards Secretariat, Acoustical Society of America, Mellive, NY

Allen JS, May DJ, Ferrara KW. 2002. Dynamics of therapeutic ultrasound contrast agents. Ultrasound in Medicine \& Biology 28:805-16

Allen TM, Cullis PR. 2004. Drug Delivery Systems: Entering the Mainstream. Science 303:1818-22

Arora M, Ohl CD. 2005. Bubble assisted drug delivery. Journal of Controlled Release 101:363-5

Bailey MR, Khokhlova VA, Sapozhnikov OA, Kargl SG, Crum LA. 2003. Physical mechanisms of the therapeutic effect of ultrasound - (A review). Acoustical Physics 49:369-88

Bao SP, Thrall BD, Miller DL. 1997. Transfection of a reporter plasmid into cultured cells by sonoporation in vitro. Ultrasound in Medicine and Biology 23:953-9

Borden MA, Kruse DE, Caskey CF, Zhao SK, Dayton PA, Ferrara KW. 2005. Influence of lipid shell physicochemical properties on ultrasound-induced microbubble destruction. Ieee Transactions on Ultrasonics Ferroelectrics and Frequency Control 52:1992-2002

Bremond N, Arora M, Ohl CD, Lohse D. 2005. Cavitation on surfaces. Journal of Physics-Condensed Matter 17:S3603-S8

Bremond N, Arora M, Ohl CD, Lohse D. 2006. Controlled multibubble surface cavitation. Physical Review Letters 96

Chen WS, Lafon C, Matula TJ, Vaezy S, Crum LA. 2003. Mechanisms of lesion formation in high intensity focused ultrasound therapy. Acoustics Research Letters Online-Arlo 4:41-6

Church CC. 2002. Spontaneous homogeneous nucleation, inertial cavitation and the safety of diagnostic ultrasound. Ultrasound in Medicine and Biology 28:1349-64

Coussios CC, Collin JRT, Muckle AJ. 2006. Non-invasive monitoring and control of inertial cavitation dynamics

during HIFU exposure in vitro. 6th International Symposium on Therapeutic Ultrasound, pp. 316-21. Oxford: American Institute of Physics

Coussios CC, Farny CH, Haar GT, Roy RA. 2007. Role of acoustic cavitation in the delivery and monitoring of cancer treatment by high-intensity focused ultrasound (HIFU). International Journal of Hyperthermia 23:105 - 20

Crum LA. 1982. NUCLEATION AND STABILIZATION OF MICRO-BUBBLES IN LIQUIDS. Applied Scientific Research 38:101-15

Crum LA, Eller AI. 1970. Motion of bubbles in a stationary sound field. Journal of the Acoustical Society of America 48:181-9

Datta S, Coussios CC, McAdory LE, Tan J, Porter T, et al. 2006. Correlation of cavitation with ultrasound enhancement of thrombolysis. Ultrasound in Medicine and Biology 32:1257-67

Devin CJ. 1959. Survey of thermal, radiation, and viscous damping of pulsating air bubbles in water. J. Acoust. Soc. Am 31:1654

Elder SA. 1959. Cavitation Microstreaming. The Journal of the Acoustical Society of America 31:54-64 
Eller A, Flynn HG. 1963. Rectified Diffusion during Nonlinear Pulsations of Cavitation Bubbles. The Journal of the Acoustical Society of America 35:1906

Eller A, Flynn HG. 1968. Generation of Subharmonics of Order One-Half by Bubbles in a Sound Field. The Journal of the Acoustical Society of America 46:722-7

Eller AI. 1974. SUBHARMONIC RESPONSE OF BUBBLES TO UNDERWATER SOUND. Journal of the Acoustical Society of America 55:871-3

Eller AI, Crum LA. 1970. Instability of motion of a pulsating bubble in a sound field. Journal of the Acoustical Society of America 47:762-7

Everbach EC, Francis CW. 2000. Cavitational mechanisms in ultrasound-accelerated thrombolysis at $1 \mathrm{MHz}$. Ultrasound in Medicine and Biology 26:1153-60

Falk MH, Issels RD. 2001. Hyperthermia in oncology. International Journal of Hyperthermia 17:1-18

Farny CH, Wu TM, Holt RG, Murray TW, Roy RA. 2005. Nucleating cavitation from laser-illuminated nano-particles. Acoustics Research Letters Online-Arlo 6:138-43

Frinking PJA, Bouakaz A, de Jong N, Ten Cate FJ, Keating S. 1998. Effect of ultrasound on the release of micro-encapsulated drugs. Ultrasonics 36:709-12

Garbin V, Cojoc D, Ferrari E, Di Fabrizio E, Overvelde MLJ, et al. 2007. Changes in microbubble dynamics near a boundary revealed by combined optical micromanipulation and high-speed imaging. Applied Physics Letters 90

Goss SA, Frizzell LA, Dunn F. 1980. Dependence of the ultrasonic properties of biological tissue. J. Acoust. Soc. Am 67:1041-4

Goss SA, Johnston RL, Dunn F. 1978a. Comprehensive compilation of empirical ultrasonic properties of mammalian tissues. The Journal of the Acoustical Society of America 64:423-57

Goss SA, Johnston RL, Dunn F. 1978b. Comprehensive compilation of empirical ultrasonic properties of mammalian tissues. J Acoust Soc Am. 64

423-47

Guzman HR, McNamara AJ, Nguyen DX, Prausnitz MR. 2003. Bioeffects caused by changes in acoustic cavitation bubble density and cell concentration: a unified explanation based on cell-to-bubble ratio and blast radius. Ultrasound in Medicine \& Biology 29:1211-22

Haar Gt, Coussios C. 2007. High intensity focused ultrasound: Physical principles and devices. International Journal of Hyperthermia 23:89 - 104

Hallow DM, Mahajan AD, McCutchen TE, Prausnitz MR. 2006. Measurement and correlation of acoustic cavitation with cellular bioeffects. Ultrasound in Medicine \& Biology 32:1111-22

Hilgenfeldt S, Lohse D. 2000. The acoustics of diagnostic microbubbles: dissipative effects and heat deposition. Ultrasonics 38:99-104

Hilgenfeldt S, Lohse D, Zomack M. 2000. Sound scattering and localized heat deposition of pulse-driven microbubbles. Journal of the Acoustical Society of America 107:3530-9

Holt RG, Roy RA. 2001. Measurements of bubble-enhanced heating from focused, MHzfrequency ultrasound in a tissue-mimicking material. Ultrasound in Medicine and Biology 27:1399-412 
Holt RG, Roy RA. 2005. Bubble dynamics in therapeutic ultrasound. In Bubble and particle dynamics in acoustic fields: : modern trends and applications, ed. A Dionikov, pp. 108-229. Kerala: Transworld Research Network

Huang J, Holt RG, Cleveland RO, Roy RA. 2004. Experimental validation of a tractable numerical model for focused ultrasound heating in flow-through tissue phantoms. Journal of the Acoustical Society of America 116:2451-8

Hynynen K, McDannold N, Sheikov NA, Jolesz FA, Vykhodtseva N. 2005. Local and reversible blood-brain barrier disruption by noninvasive focused ultrasound at frequencies suitable for trans-skull sonications. Neuroimage 24:12-20

Jang SH, Wientjes MG, Lu D, Au JLS. 2003. Drug Delivery and Transport to Solid Tumors. Pharmaceutical Research 20:1337-50

Jeffers J, Feng RQ, Fowlkes JB, Brenner DE, Cain CA. 1991. Sonodynamic therapy: activation of anticancer agents withultrasound. Ultrasonics Symposium, 1991. Proceedings., IEEE 1991:1367-70

Kamath V, Prosperetti A. 1989. NUMERICAL-INTEGRATION METHODS IN GASBUBBLE DYNAMICS. Journal of the Acoustical Society of America 85:1538-48

Keller JB, Miksis M. 1980. Bubble oscillations of large amplitude. The Journal of the Acoustical Society of America 68:628

Kennedy JE. 2005. High-intensity focused ultrasound in the treatment of solid tumours. Nat Rev Cancer 5:321-7

Khokhlova VA, Bailey MR, Reed JA, Cunitz BW, Kaczkowski PJ, Crum LA. 2006. Effects of nonlinear propagation, cavitation, and boiling in lesion formation by high intensity focused ultrasound in a gel phantom. Journal of the Acoustical Society of America 119:1834-48

Kinoshita M, Hynynen K. 2006. Mechanism of porphyrin-induced sonodynamic effect: Possible role of hyperthermia. Radiation Research 165:299-306

Lafon C, Zderic V, Noble ML, Yuen JC, Kaczkowski PJ, et al. 2005. Gel phantom for use in high-intensity focused ultrasound dosimetry. Ultrasound in Medicine and Biology 31:1383-9

Lauer CG, Burge R, Tang DB, Bass BG, Gomez ER, Alving BM. 1992. Effect of ultrasound on tissue-type plasminogen activator-induced thrombolysis. Circulation 86:1257-64

Leighton TG. 1994. The Acoustic Bubble: Academic Press

Leighton TG. 1997. A strategy for the development and standardisation of measurement methods for high power/cavitating ultrasonic fields: review of cavitation monitoring techniques, University of Southampton - Institute of Sound and Vibration Research

Leighton TG, Phelps AD. 1997. The mechanism of subharmonic ultrasound modulation by forcibly oscillating bubbles. Ultrasonics 35:183-

Leslie TA, Kennedy JE. 2007. High intensity focused ultrasound in the treatment of abdominal and gynaecological diseases. International Journal of Hyperthermia 23:173 - 82

Liu RH, Hang J, Pindera MZ, Athavale M, Grodzinski P. 2002. Bubble-induced acoustic micromixing. Lab Chip 2:151-7

Longuet-Higgins MS. 1990. Bubble noise spectra. The Journal of the Acoustical Society of America 87:652-61 
Longuet-Higgins MS. 2006. Monopole emission of sound by asymmetric bubble oscillations. Part 1. Normal modes. Journal of Fluid Mechanics Digital Archive 201:525-41

Maksimov AO, Leighton TG. 2001. Transient processes near the acoustic threshold of parametrically-driven bubble shape oscillations. Acustica 87:322-32

Marin A, Muniruzzaman M, Rapoport N. 2001. Mechanism of the ultrasonic activation of micellar drug delivery. Journal of Controlled Release 75:69-81

Marmottant P, van der Meer S, Emmer M, Versluis M, de Jong N, et al. 2005. A model for large amplitude oscillations of coated bubbles accounting for buckling and rupture. Journal of the Acoustical Society of America 118:3499-505

McDannold N, Vykhodtseva N, Hynynen K. 2006. Targeted disruption of the blood-brain barrier with focused ultrasound: association with cavitation activity. Physics in Medicine and Biology 51:793-807

McPherson DD, Holland CK. 2003. Seizing the science of ultrasound - Beyond imaging and into physiology and therapeutics. Journal of the American College of Cardiology 41:1628-30

Mesiwala AH, Farrell L, Wenzel HJ, Silbergeld DL, Crum LA, et al. 2002. Highintensity focused ultrasound selectively disrupts the blood-brain barrier in vivo. Ultrasound in Medicine and Biology 28:389-400

Miller DL, Kripfgans OD, Fowlkes JB, Carson PL. 2000. Cavitation nucleation agents for nonthermal ultrasound therapy. Journal of the Acoustical Society of America 107:3480-6

Miller DL, Thomas RM. 1995. ULTRASOUND CONTRAST AGENTS NUCLEATE INERTIAL CAVITATION IN-VITRO. Ultrasound in Medicine and Biology 21:1059-65

Molema G, de Leij L, Meijer DKF. 1997. Tumor Vascular Endothelium: Barrier or Target in Tumor Directed Drug Delivery and Immunotherapy. Pharmaceutical Research 14:2-10

Munshi N, Rapoport N, Pitt WG. 1997. Ultrasonic activated drug delivery from pluronic P-105 micelles. Cancer Letters 118:13-9

Neppiras EA, Noltingk BE. 1951. Cavitation produced by ultrasonics: theoretical conditions for the onset of cavitation. Proc. Phys. Soc. B64:1032-8

Nyborg WL. 1965. Acoustic streaming. Physical Acoustics 2:65-331

Ohl CD, Arora M, Ikink R, de Jong N, Versluis M, et al. 2006. Sonoporation from jetting cavitation bubbles. Biophysical Journal 91:4285-95

Parsons JE, Cain CA, Abrams GD, Fowlkes JB. 2006. Pulsed cavitational ultrasound therapy for controlled tissue homogenization. Ultrasound in Medicine and Biology 32:115-29

Pennes HH. 1948. Analysis of Tissue and Arterial Blood Temperatures in the Resting Human Forearm. Journal of Applied Physiology 1:93-122

Pierce AD. 1981. Acoustics: an introduction to its physical principles and applications: McGraw-Hill Book Company

Prentice P, Cuschieri A, Dholakia K, Prausnitz M, Campbell P. 2005. Membrane disruption by optically controlled microbubble cavitation. Nat Phys 1:107-10

Prokop AF, Soltani A, Roy RA. 2007. Cavitational Mechanisms in UltrasoundAccelerated Fibrinolysis. Ultrasound in Medicine \& Biology 33:924-33 
Prosperetti A. 1975. NONLINEAR OSCILLATIONS OF GAS-BUBBLES IN LIQUIDS - TRANSIENT SOLUTIONS AND CONNECTION BETWEEN SUBHARMONIC SIGNAL AND CAVITATION. Journal of the Acoustical Society of America 57:810-21

Prosperetti A. 1977. THERMAL EFFECTS AND DAMPING MECHANISMS IN FORCED RADIAL OSCILLATIONS OF GAS-BUBBLES IN LIQUIDS. Journal of the Acoustical Society of America 61:17-27

Prosperetti A, Crum LA, Commander KW. 1988. NONLINEAR BUBBLE DYNAMICS. Journal of the Acoustical Society of America 83:502-14

Rabkin BA, Zderic V, Vaezy S. 2005. Hyperecho in ultrasound images of HIFU therapy: Involvement of cavitation. Ultrasound in Medicine and Biology 31:947-56

Rapoport N, Gao Z, Kennedy A. 2007. Multifunctional Nanoparticles for Combining Ultrasonic Tumor Imaging and Targeted Chemotherapy. J. Natl. Cancer Inst.:djm043

Rivens I, Shaw A, Civale J, Morris H. 2007. Treatment monitoring and thermometry for therapeutic focused ultrasound. International Journal of Hyperthermia 23:121 39

Roberts WW, Hall TL, Ives K, Wolf JS, Fowlkes JB, Cain CA. 2006. Pulsed cavitational ultrasound: A noninvasive technology for controlled tissue ablation (histotripsy) in the rabbit kidney. Journal of Urology 175:734-8

Schlicher RK, Radhakrishna H, Tolentino TP, Apkarian RP, Zarnitsyn V, Prausnitz MR. 2006. Mechanism of intracellular delivery by acoustic cavitation. Ultrasound in Medicine \& Biology 32:915-24

Sokka SD, Gauthier TP, Hynynen K. 2005. Theoretical and experimental validation of a dual-frequency excitation method for spatial control of cavitation. Physics in Medicine and Biology 50:2167-79

Sokka SD, King R, Hynynen K. 2003. MRI-guided gas bubble enhanced ultrasound heating in in vivo rabbit thigh. Physics in Medicine and Biology 48:223-41

Stride E, Saffari N. 2003. On the destruction of microbubble ultrasound contrast agents. Ultrasound in Medicine and Biology 29:563-73

Tartis MS, McCallan J, Lum AFH, LaBell R, Stieger SM, et al. 2006. Therapeutic effects of paclitaxel-containing ultrasound contrast agents. Ultrasound in Medicine \& Biology 32:1771-80

Tho P, Manasseh R, Ooi A. 2007. Cavitation microstreaming patterns in single and multiple bubble systems. Journal of Fluid Mechanics 576:191-233

Thomas CR, Farny CH, Coussios CC, Roy RA, Holt RG. 2005. Dynamics and control of cavitation during high-intensity focused ultrasound application. Acoustics Research Letters Online-Arlo 6:182-7

Tran BC, Seo J, Hall TL, Fowlkes JB, Cain CA. 2003. Microbubble-enhanced cavitation for noninvasive ultrasound surgery. Ieee Transactions on Ultrasonics Ferroelectrics and Frequency Control 50:1296-304

Trubestein G, Engel C, Etzel F, Sobbe A, Cremer H, Stumpff U. 1976. Thrombolysis by ultrasound. Clin Sci Mol Med Suppl 3:697s-8s

Tu J, Hwang JH, Matula TJ, Brayman AA, Crum LA. 2006a. Intravascular inertial cavitation activity detection and quantification in vivo with optison. Ultrasound in Medicine and Biology 32:1601-9 
Tu J, Matula TJ, Brayman AA, Crum LA. 2006b. Inertial cavitation dose produced in ex vivo rabbit ear arteries with Optison (R) by 1-MHz pulsed ultrasound. Ultrasound in Medicine and Biology 32:281-8

Vaezy S, Zderic V. 2007. Hemorrhage control using high intensity focused ultrasound. International Journal of Hyperthermia 23:203 - 11

van Wamel A, Bouakaz A, Bernard B, ten Cate F, de Jong N. 2005. Controlled drug delivery with ultrasound and gas microbubbles. Journal of Controlled Release 101:389-91

van Wamel A, Kooiman K, Harteveld M, Emmer M, ten Cate FJ, et al. 2006. Vibrating microbubbles poking individual cells: Drug transfer into cells via sonoporation. Journal of Controlled Release 112:149-55

Vykhodtseva NI, Hynynen K, Damianou C. 1995. HISTOLOGIC EFFECTS OF HIGHINTENSITY PULSED ULTRASOUND EXPOSURE WITH SUBHARMONIC EMISSION IN RABBIT BRAIN IN-VIVO. Ultrasound in Medicine and Biology 21:969-79

Wu J, Chappelow J, Yang J, Weimann L. 1998. Defects generated in human stratum corneum specimens by ultrasound. Ultrasound in Medicine \& Biology 24:705-10

$\mathrm{Xu}$ Z, Fowlkes JB, Cain CA. 2006. A new strategy to enhance cavitational tissue erosion using a high-intensity, initiating sequence. Ieee Transactions on Ultrasonics Ferroelectrics and Frequency Control 53:1412-24

Xu Z, Fowlkes JB, Ludomirsky A, Cain CA. 2005. Investigation of intensity thresholds for ultrasound tissue erosion. Ultrasound in Medicine and Biology 31:1673-82

Yang X, Church CC. 2005. A model for the dynamics of gas bubbles in soft tissue. The Journal of the Acoustical Society of America 118:3595-606

Yang XM, Church CC. 2006. A simple viscoelastic model for soft tissues in the frequency range 6-20 MHz. Ieee Transactions on Ultrasonics Ferroelectrics and Frequency Control 53:1404-11

Yang XM, Roy RA, Holt RG. 2004. Bubble dynamics and size distributions during focused ultrasound insonation. Journal of the Acoustical Society of America $116: 3423-31$ 